\title{
LES RACES DE L'ABEILLE DOMESTIQUE EN ITALIE ET LEUR DISTRIBUTION GÉOGRAPHIQUE
}

\author{
PAR
}

\section{Delia GUIGLIA}

Museo civico di storia naturale Giacomo Doria, GENOVA (Italie)

Même dans les plus anciens ouvrages, tels ceux d'Aristote et de Virgile, on parle déjà de l'existence en Italie de deux variétés d'abeilles : les abeilles noires et les abeilles dorées. Plusieurs auteurs par la suite discutèrent sur l'existence supposée d'autres variétés. G. B. Grassi (r88I), en sa monographie sur les abeilles d'Italie dit : "Le varietà di queste nostre api, sono tante in uno stesso paese, anzi in uno stesso apiario e talvolta perfino in uno stesso alveare; che, a voler dare un giudizio esatto, è mestieri recarci sul luogo e studiare molte e molte popolazioni, affine di poter rilevare se e quali differenze meritino considerazione, o meno " (I)

Aujourd'hui il est certain qu'en Italie sont présentes quatre races d'abeilles: Apis mellifera-mellifera $\mathrm{I}_{\text {. }}$; Apis mellifera-carnica Pol. ; Apis mellifera-ligustica Spin.; Abeille noire de Sicile.

De celles-ci 1'A pis mellifera-ligustica est l'abeille italienne proprement dite et elle est entre toutes la plus recherchée. Elle diffère de la melliferamellifera ou abeille allemande, avec l'abdomen complètement noir, parce que l'ouvrière (la couleur de la reine et des mâles est assez variable) a des bandes jaune-or ou jaune-orange sur les premiers segments de 1'abdomen.

Sa supériorité sur toutes les autres était déjà reconnue par les agriculteurs de l'ancienne Rome : Virgile, après avoir parlé des abeilles noires, élève un hymne à celles qui " brillent et luisent comme l'or ", comme étant de la race la meilleure.

L'abeille italienne est désormais répandue dans le monde entier, et les apiculteurs étrangers, trouvant réunies en elle toutes les meilleures qualités, l'ont aussitôt préférée, aidant à sa diffusion dans les pays les plus lointains. Ses mérites sont très nombreux et de grande importance

(1) I.es variétés de nos abeilles sont nombreuses non seulement dans la même région mais aussi dans un même élevage et parfois dans une même ruche; de telle façon que, si l'on veut juger exactement, il est nécessaire de se porter sur place et d'étudier de très nombreux exemplaires afin de pouvoir remarquer quels caractères sont différents et quels autres ne le sont pas. 
pour une bonne réussite de l'élevage. Elle supporte très bien le froid et en général les maladies n’ont pas de prise sur elle : en effet, entre toutes, les races italiennes, c'est celle qui résiste le mieux à la loque européenne, et dans les régions où cette maladie est répandue on cherche à se défendre en introduisant des reines italiennes dans chaque ruche. Une autre qualité de notre abeille est la propreté : sa ruche est en général très propre, et il est très difficile d'y trouver des mites, puisque l'abeille italienne sait bien se défendre de ses ennemis.

Elle a peu ou point de tendance à l'essaimage, sait régler sa ponte selon la quantité du nectar disponible et elle limite l'élevage du couvain même dès que celui-ci risque de ne pas suffire aux besoins de la ruche.

Elle est active et elle a un caractère assez pacifique, devenant agressive seulement si elle est dérangée. Elle est en général moins prolifique que les races orientales, mais toujours plus prolifique que 1'abeille noire; il ne faut pas d'autre part oublier que notre reine a une vie plus longue que celle des reines des autres races.

En raison de toutes ces qualités 1'abeille italienne a été exportée dans le monde entier et en Italie on a aujourd'hui des élevages spécialisés de reines d'abeille qui sont connus un peu partout.

La supériorité économique de l'abeille italienne a rendu nécessaire une étude sur la distribution géographique de cette précieuse race dans notre Péninsule, afin de pouvoir établir en quels points elle se trouve à l'état pur, dans quels autres à l'état hybride, afin de prévenir des croisements éventuels ou intervenir avec des œuvres de sélection.

La Prof. A. VEcchi s'était à plusieurs reprises occupée de ce problème, (r927, r940), en signalant les points où prédomine la melliferaligustica, la mellifera-mellifera et la forme hybride. Mes recherches à ce propos m'ont permis d'ajouter à ceux-ci d'autres points où prédomine l'abeille noire. J'ai ainsi remarqué sa présence en de nombreux points de la Vallée d'Aoste (Valsavaranche ; Saint Vincent; Aoste ; Courmayeur ; Petit Saint-Bernard; Cheneil ; Valtournanche). De plus, en cette dernière localité $j$ 'ai pu constater que la variété noire est bien plus nombreuse que la variété ligustica. Ce fait pourrait faire penser que dans la Vallée d'Aoste 1'abeille noire a été la vraie abeille indigène, et que par la suite une immigration spontanée ou une importation par la main de l'homme y ont apporté la ligustica.

Pour ce qui concerne la Ligurie j'ai pu voir l'abeille noire en plusieurs points de la côte à l'ouest de Gênes (Albenga ; Borgio-Verezzi ; Finale Marina ; Savona ; Albisola ; Varazze; Santuario di N. S. della Vittoria; Sarissola, Apennin Ligurien aux environs de Gênes; Gênes); ce fait démontre donc qu'elle ne vit pas seulement dans la zone la plus occidentale de la Ligurie, mais aussi, mêlée à la ligustica, le long de toute la côte, jusqu'à Gênes, pour disparaître tout à fait dans la Ligurie orientale. 
J'ai trouvé l'abeille noire mélangée à la ligustica même sur l'île de Capraia. En Sicile, selon ce que j'ai pu constater après l'examen de nombreux matériel de différentes localités (Casteldaccia, Palermo; Comune di Motta S. Anastasia, Belpasso, Catania), elle dépasse de beaucoup la variété ligustica, laquelle, comme soutient GrassI (1. c.) n'est jamais de "sangue

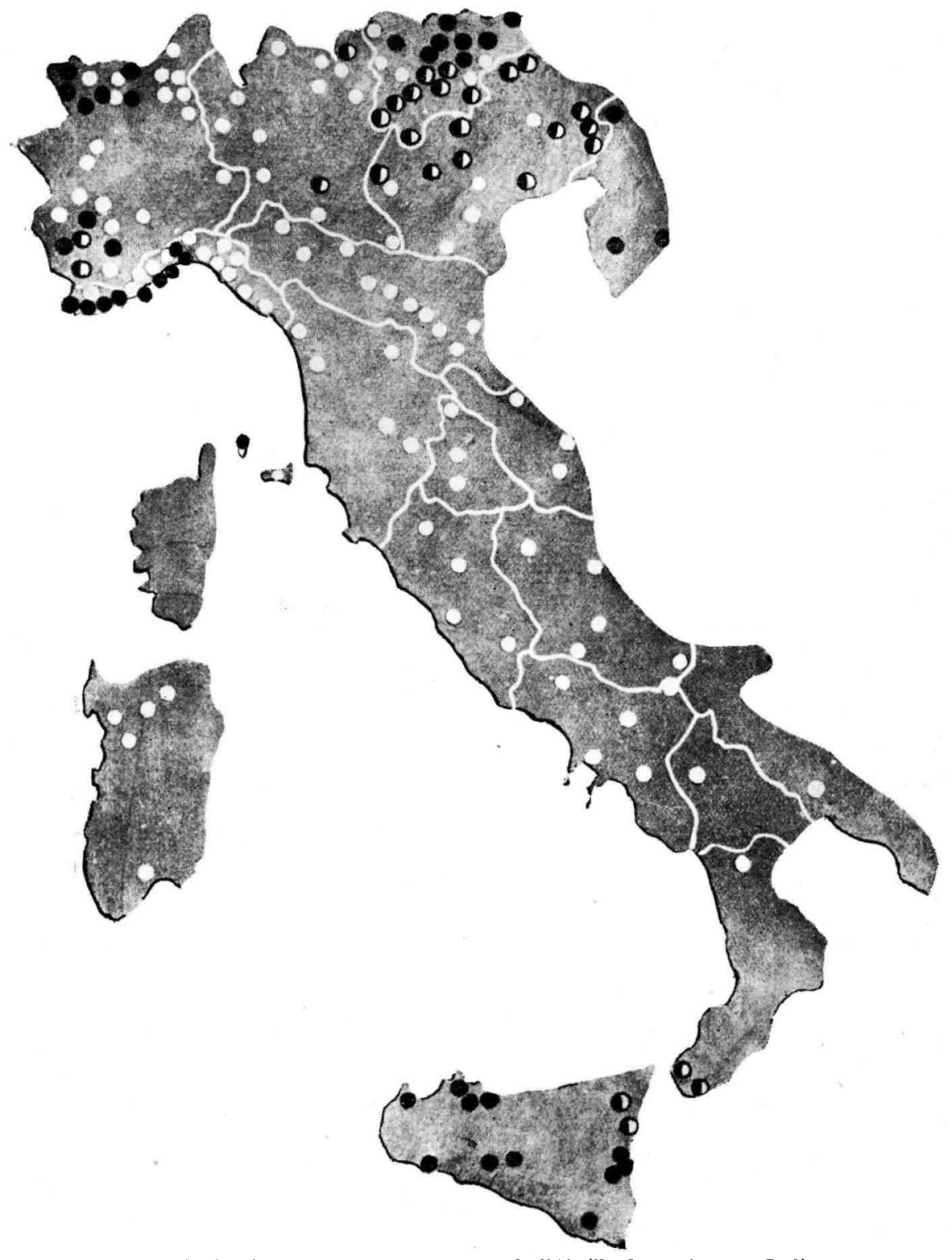

La distribution géographique des races de l'Abeille domestique en Italie (d'apres Vecchi, avec nouvelles signalisations).
Apis mellifera ligustica $S p$.
- Apis mellifera-mellifera $I_{\text {. }}$
- Api ibride 
purissimo " ('). Il est probable qu'elle est d'immigration ou d'importation. récente tandis que la vraie abeille indigène devrait être l'abeille noire, appelée "Ape di Sicilia " (2) et en terme dialectal "Ape picciridda" $\left.{ }^{3}\right)$. Elle diffère de la ligustica non seulement par la couleur complètement noire du dermasquelette de l'abdomen, mais aussi, selon différents auteurs, par ses dimensions un peu inférieures.

Ce dernier caractère, ainsi que dit S. Trajna (I933), et ainsi que $j$ 'ai pu moi-même constater par l'examen de quelques populations, est peu évident, ou mieux, comme dit Trajna, "1'Ape di Sicilia " sembrerebbe un tantino più grande » ( $\left.{ }^{4}\right)$. Cet auteur (1. c. p. I2) dit encore : "Importanti pure sono gli orli bianchicci delle tibie delle prime due paia di zampe dell' A. ligustica contro l'unico del $2^{\circ}$ paio dell' $A$. di Sicilia, l'orlo rossiccio del primo articolo tarsale del $3^{\circ}$ paio di zampe dell' $A$. di Sicilia, mancante nell' $A$. ligustica e il il contrasto degli uncini neri col tarso rossiccio nelle zampe dell' $A$. ligustica " $\left({ }^{5}\right)$. Ces caractères ne sont toutefois pas toujours facilement appréciables.

Sur ses origines et sur son ascendance nous sommes encore très incertains. Il y a des auteurs qui la relient à la mellifera-mellifera de l'Europe du Nord, des autres qui la font dériver de 1'abeille de Dalmatie, et des autres encore qui la font naitre d'un croisement de la ligustica avec la cecropia. Je serais pour ma part de l'avis, comme j'ai déjà dit (I953,p.25), de la rapprocher à l'abeille noire de l'Afrique du Nord (A.m. intermissa Butt. Reep.) ; en effet, en comparant des individus de Tunis avec des autres de différentes localités de la Sicile, je n'ai pas trouvé une remarquable différence de caractères. Je me propose de reprendre la question lorsque je pourrai étudier sur place les abeilles vraiment indigènes, c'est-à-dire pas encore corrompues par de probables infiltrations d'éléments étrangers.

\section{RÉFÉRENCES BIBLIOGRAPHIQUES}

Grassi (B.). - Saggio di una Monografia delle Api d'Italia. L'apicultore $\mathrm{n}^{\mathrm{0}} \mathrm{g}, \mathbf{I} 88 \mathrm{I}$.

Guiglia (D.). - Ricerche zoologiche sul Massiccio del Pollino (LucaniaCalabria). Ann. Istit. Zool. Univ. Napoli, V, 110 9, I953.

Trajna (S.). - L'Ape nera di Sicilia in confronto della ligustica. Nota preventiva. Bull. Ist. Zool. Univ. Palermo, II, fasc. 4-5, r933.

VECCHI (A.). - Sulla distribuzione geografica dell'Apis mellifica ligustica Spin. Boll. Labor. Zool. gen. e agraria, Portici, XX, p. I50-168, 1927. VECCHI (A.). - Il primo decennio di vita dell'Istituto Nazionale di Apicoltura. Tipogr. Compositori, Bologna, p. 3-15, I940.

(1) "Sangue purissimo " = sang tout à fait pur.

(2) "Ape di Sicilia " = Abeille de Sicile.

(3) "Ape picciridda " = Abeille petite.

(4) "L'Abeille de Sicile semblerait un peu plus grande".

( 5 ? "Importants sont aussi les bords blanchâtres des deux premières paires de pattes de l'A. ligusttica à l'égard de la seule seconde paire de l'A. di Sicilia, le bord rougeâtre du premier article du tarse de la $3^{\mathrm{e}}$ paire de pattes de l'A. di Sicilia qui manque dans l'. A. ligustica, et le contraste entre les crochets noirs et le tarse rougeátre dans les pattes de l'A. ligusisica. 